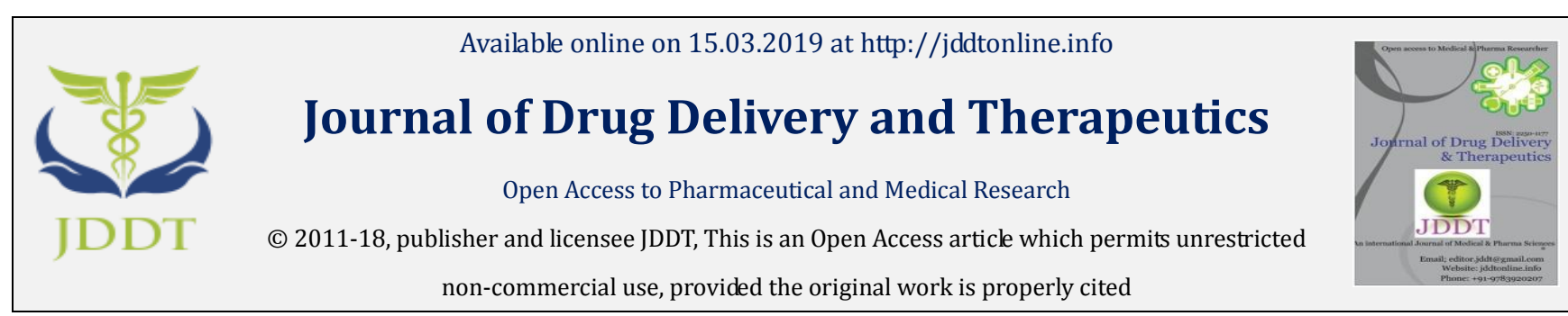

Open 2 Access

Mini Review

\title{
The ancient, Present and Future Prospects of Tongue Manifestation
}

\author{
Yu-Hui Ma' ${ }^{1}$, Lin-Feng Li ${ }^{1}$, Hao-Nan Wu${ }^{1}$, Yu-Xuan Zhang' ${ }^{2}$, Yu Zhang ${ }^{3}$, Yu-Rong Liang*1 \\ ${ }^{1}$ College of Chinese Medicine, Hebei University, Baoding, Hebei province, China. \\ ${ }^{2}$ College of Cyberspace Security and Computer Science, Hebei University, Baoding, Hebei province, China. \\ ${ }^{3}$ College of Medicine, Hebei University, Baoding, Hebei province, China.
}

\begin{abstract}
Tongue inspection with a long history is one of the most distinctive methods in diagnosis of Traditional Chinese Medicine (TCM). The emergence and improvement of tongue inspection theory is the crystallization of countless ancient medical doctors' wisdom. We should combine the theory of tongue inspection and modern technology and interchange TCM and western medicine, which is conducive to clinical research and promotes the objective development of tongue inspection, achieving the inheritance of TCM and creating TCM of our time.
\end{abstract}

Keywords: tongue inspection; ancient; modern; future prospects

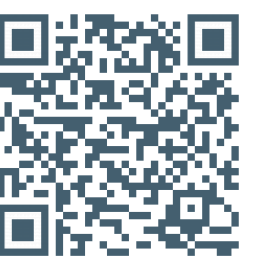

\section{Cite this article as:}

Ma Y, Li L, Wu H, Zhang Y, Zhang Y, Liang Y, The ancient, Present and Future Prospects of Tongue Manifestation, Journal of Drug Delivery and Therapeutics. 2019; 9(2):409-414 http://dx.doi.org/10.22270/jddt.v9i2.2423

*Address for Correspondence:

Yu-Rong Liang, College of Chinese Medicine, Hebei University, Baoding, Hebei province, China.

\section{Introduction}

The four diagnostic methods of TCM are "Looking, listening, asking and feeling the pulse", tongue inspection as a part of the diagnosis, has a significant place in clinical diagnosis. The tongue inspection has a long history. According to the relevant data 1 , the earliest documentary about the tongue Chinese medicine history appeared in the Yin and Shang Dynasties. It sprouted in the Western Zhou Dynasty and the Spring and Autumn Period, and the theory was perfected and developed during the Song, Jin and Yuan Dynasties. With the prosperity of economy and culture of Ming and Qing Dynasties, the development of tongue inspection reached the peak. With the liberation and establishment of New China, the development of tongue inspection opened a new chapter. In the 21st century, with the rapid development of economy and productivity and the high-frequency renewal of science and technology, human beings have entered into a high-speed era; the development of tongue inspection is facing a huge reform, which is that the trend of digitization and objectification is inevitable. As modern people, we should sum up the essence of the ancients so that tongue inspection can be applied to clinical practice, and we should collect the clinical cases and summarize the theory of tongue inspection for creating the tongue inspection of our own time. Only in this way, can tongue inspection come from clinical practice and serve clinical practice as well. 


\section{The meaning and the function of tongue inspection}

\subsection{Meaning}

Tongue inspection, a word of TCM diagnostics, is a method of assisting diagnosis and differentiating diseases by observing the changes of tongue quality (spirit, color, shape, condition), fur (character, color) and sublingual vessel (shape, color). The tongue is the opening of the heart, the outer part of the spleen, and the fur is fumigated by stomach qi. The state of the tongue can reflect the physiological and pathological state of five Zang-viscera and six Fu-viscera, which has a certain distribution law. The tip of tongue reflects the pathological changes of the heart and lung, the middle surface of tongue reflects the spleen and stomach, and the root of tongue reflects the kidney, and the liver and gallbladder are on both sides of the tongue (Fig.1). In addition, the tongue and five Zang-viscera are connected by meridians and collaterals, the Collateral of Heart Meridian is tied to tongue, the Spleen Meridian of Foot-Taiyin is connected with tongue and scattered under tongue, the Kidney Channel of Foot-Shaoyin extends along both side of the root of tongue, the Liver Channel of Foot-Jueyin connects the root of the tongue, all of which illustrate that tongue manifestation can be connected with five Zang-viscera and six Fu-viscera through meridians and veins directly or indirectly. The change of the state of essence, qi, blood and fluid in Zang-Fu viscera can be expressed by tongue manifestation, and accordingly, it can reflect the excess and deficiency of Zang-Fu viscera, the rising and falling of essence, qi, blood and body fluid, and the nature of the disease, the depth of the disease, which can help to make the corresponding judgment ${ }^{2}$.

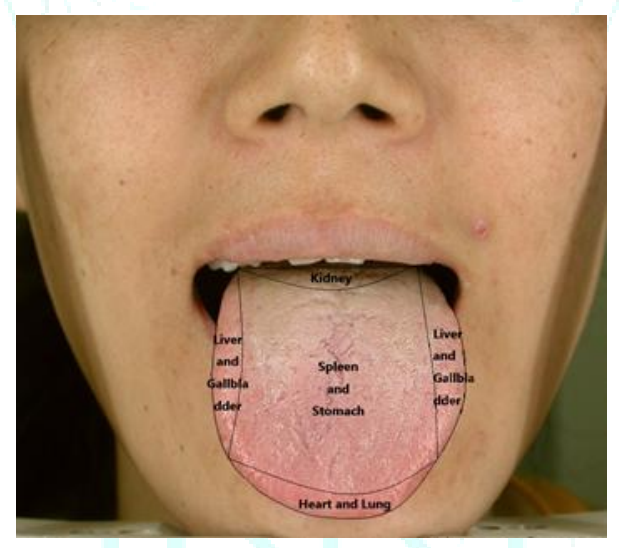

\section{Figure1: Distribution of viscera on tongue}

\subsection{Function}

\subsubsection{Judging constitution according to tongue manifestation}

According to nine constitutions of TCM ${ }^{3}$, we can classify and summarize the constitution according to tongue manifestation. Moderate quality: the tongue manifestation is normal, pink tongue with thin white fur, the tongue shape and quality is normal, no abnormal tongue shape; Qi deficiency: the tongue color is pink or pale, the fur is in moderate size and wet but not sticky, tender tongue, no abnormal tongue shape, it might be plump tongue or teeth-printed tongue; Yang deficiency: the tongue color is pale or light purple, the tongue is fat and big with teeth marks, tender tongue, the fur is thin and moist, no abnormal tongue condition; Yin deficiency: it is red or deep red tongue with a little fur which might be eroded fur even mirror-like tongue, and it is pretty dry; Phlegm and Dampness: it is plump tongue with thick greasy white fur; Damp Heat: it is red tongue with greasy fur; Blood Stasis: light purple tongue or purple tongue with blood stasis spots, its sublingual vessel gets thickened and varicose whose color is cyanotic or dark purple; Qi stagnancy: pink tongue, slightly thick white fur and it might be greasy or dry; Specific Intrinsic Qualities: the tongue manifestation shows different characteristics depending on the disease. (Table 1) 
Table 1: The tongue manifestation of nine constitutions of TCM

\begin{tabular}{|l|l|}
\hline moderate quality & pink tongue with thin white fur \\
\hline qi deficiency & $\begin{array}{l}\text { pink but tender tongue or pale but tender tongue or tender tongue with teeth marks or plump } \\
\text { tongue }\end{array}$ \\
\hline yang deficiency & pale or light purple tongue, plump tender tongue with teeth marks \\
\hline yin deficiency & red tongue, eroded tongue, mirror-like tongue \\
\hline phlegm damp & plump tongue, greasy thick white fur \\
\hline damp heat & red tongue, greasy yellow fur \\
\hline blood stasis & $\begin{array}{l}\text { light purple tongue or purple tongue with blood stasis spots, thickened and varicose cyanotic or } \\
\text { dark purple sublingual vessel }\end{array}$ \\
\hline qi stagnancy & thick dry white fur \\
\hline specific intrinsic qualities & the tongue manifestation shows different characteristics depending on the disease \\
\hline
\end{tabular}

\subsubsection{Diagnosis and prognosis are based on tongue}

By observing tongue manifestation, doctors can know the cause, location and prognosis of the disease. In ancient time, Hua Tuo said "If Upper-Jiao is excess syndrome, the sweat will spring up on forehead, but torso will not perspire, the patient can eat and breathe and his tongue is dry." Zhong Jing said "What is binding of Zang-viscera? Answer: such as chest binding syndrome...... Guan Pulse is thin and tight, which is called binding of Zang-viscera. The patient whose fur is white is difficult to be cured. "Sun Simiao said, "Visceral heat causes sores on the tongue and leads to red lips; if viscera is cold, the tongue will shrink and the lips will be green, so the cold should be supplemented, the heat should be purged, if it is nether cold nor hot, we should treat according to the viscera "; Nowadays, many excellent scholars have done many in-depth researches in this field. Shen Shaogong ${ }^{4}$ believes that" The tongue is a barometer of changes in human organs". They put forward that tongue inspection can be divided into three steps: the inspection of fur, the inspection of tongue quality and the inspection of corpus linguae, and concludes that coldness or heat is depended on the color of fur, excess and deficiency is depended on the thickness of fur, the adequacy and deficiency of fluid is depended on the moistening dryness of fur, the position of disease is determined by the position of fur. Wang Chunrong ${ }^{5}$ thinks that tongue inspection can contribute to treatment based on syndrome differentiation then help for clinical medication. For example, the causes of eroded tongue in children mostly own to the deficiency of Yin of spleen and stomach or syndrome of consumption of fluid because of the long-term heat. If the tongue quality is pale and the fur is thin and white, it is mostly due to the deficiency of Yin of spleen and stomach. Dendrobium and fragrant solomonseal rhizome should be used in clinic to nourish Yin of spleen and stomach. If the tongue is red and the fur is thick greasy and peeling, which is the result of long-term heat for food retention syndrome, Baohe Pill with Dendrobium and Ophiopogon japonicus should be used to clear heat and remove food stagnation. Professor Wang Yanhui 6 has accumulated a lot of clinical experience through long-term treatment of exogenous damp-heat disease. He believes that doctors should focus on tongue when they treat exogenous damp-heat disease, which can help doctors deal with the relationship between eliminating pathogen and strengthening vital qi.

\section{Tongue diagnosis in ancient}

Ancient doctors made a great contribution to the emergence, enrichment and perfection of the tongue inspection theory. As a part of Chinese traditional culture, it is the crystallization of the wisdom and long-term practice of the ancient working people. Tongue inspection has a long history. The earliest records of it appeared in the Yin and Shang Dynasties, after that more medical works such as Huangdi Neijing, Shanghan Lun and Qianjin Fang were created. The tongue inspection theory has gradually developed and enriched with time. Huangdi Neijing, the earliest classical medical book in China, has recorded the relevant records about tongue diagnosis, which has constructed the rudiment of tongue inspection theory system. Zhang Zhongjing who lived in the Eastern Han 
Dynasty first used the word "tongue foetus" and supplemented the observation of tongue condition, tongue sense, tongue quality and fur. The book he wrote, called "Shanghan Zabing Lun", combined tongue inspection with syndrome differentiation of six channels theory and syndrome differentiation of Zang-fu viscera, which laid a foundation for the application of tongue inspection in science of epidemic febrile disease of TCM. Zhu Bing Yuan Hou Lun of Sui Dynasty's refers to the inspection of corpus linguae and tongue color as well as sublingual vessel, which said that corpus linguae can be divided into: glossoncus, contracted tongue, frequent protrusion of tongue, double tongue, etc. The tongue color can be divided into: green tongue, red tongue, yellow, white, black tongue, etc. ${ }^{9}$. By the Song, Jin and Yuan Dynasties, the theory of tongue inspection had become systematic after the sublimation of previous experience. The first monograph of tongue inspection, Ao Shi Shang Han JIn

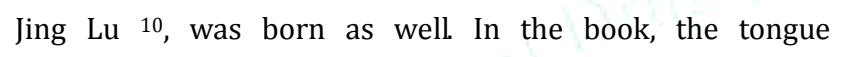
manifestation was used to distinguish the nature of the disease, and the principle of "using medicine according to tongue manifestation" was formulated, and the idea that tongue manifestation can be used for prognosis was permeated.

\section{Tongue diagnosis in modern}

Modern tongue inspection is rooted in the theory of the ancients. By using modern science and technology, it combines tongue inspection theory researches of TCM with western medicine in order to make it more objective and meticulous.

\subsection{Research Indicators towards Objectification}

In ancient, doctors observed the tongue quality (spirit, color, shape, condition), fur (character, color) and sublingual vessel (shape, color) through their eyes. It was susceptible to the subjective factors and objective factors like environment. Therefore, in order to adapt to modern research, with the help of computer image processing technology and digital camera equipment, tongue inspection has begun to digitize and objectify since 1980 's 11 . Shiqiang et al. 12 compared the characteristics of traditional tongue image observation light and artificial light source then analyzed the result. It was concluded that the color temperature of standard light source D50 was close to the lighting environment of traditional tongue manifestation observation. The conclusion provided a feasible solution to the light problem when doctors collect tongue manifestation information. In tongue color analysis, $\mathrm{Li}$ Bocong et al. 13 used CIELAB uniform color space platform technology (Fig. 2, Fig. 3) to combine color science theory with tongue diagnosis analysis, which made it feasible to objectify the indexes of tongue color. In the characteristics of fur and tongue quality, Shen Lansun et al. ${ }^{14}$ used Snakes Model of Catmull-Rom Spline Function, Greedy algorithm and Support Vector Machine to segment tongue image and analyze the characteristic of fur and tongue quality. At the same time, a multi-scale edge detection method is proposed to analyze the tongue image cracks.



Figure 2: $\mathrm{A}^{*}-\mathrm{b}^{*}$ Planar Projection of Sample Points of Tongue Colors

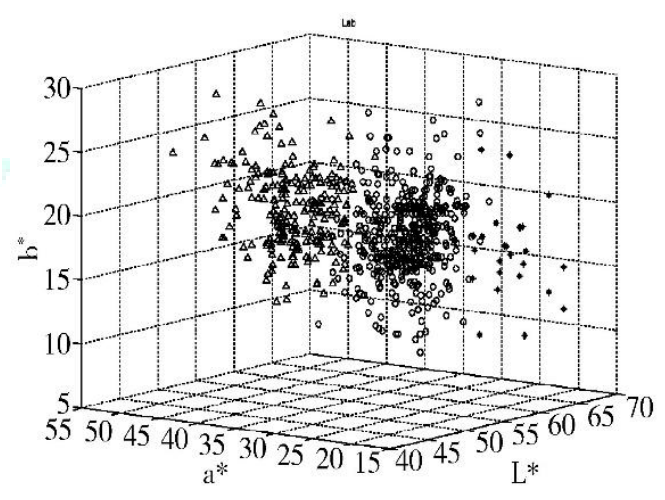

Figure 3: Three-dimensional distribution maps of various tongue colors

\subsection{Meticulousness of Research Indicators}

In ancient, the doctors used comprehensive analysis of data gained by four diagnostic methods and treatment based on syndrome differentiation to treat patients. Nowadays, traditional Chinese medicine is mainly based on diagnostic methods and theories of traditional Chinese medicine, supplemented by Western medical technology. On the basis of the four diagnostic methods, it combines the Western medical 
indicators of patients in order to achieve better therapeutic effect. Many doctors and researchers combine digital tongue manifestation with Western medicine's laboratory indicators to summarize and analyze the relationship, which enriches the theory of tongue diagnosis in TCM and greatly promotes the development of tongue diagnosis in TCM. Xu Jie et al. 15 analyzed tongue image and blood sugar indexes of diabetic patients with tongue diagnostic instrument, concluding that HbA1c was negatively correlated with tongue color parameters $\mathrm{a}, \mathrm{b}$ and fur texture parameters ASM, positively correlated with tongue quality, fur texture parameters L and tongue quality texture parameters CON, ENT, MEAN, and negatively correlated with fasting blood sugar (FBG) and tongue color parameters a, and fur texture parameters $a$. The color parameter L was positively correlated with the fur parameter but not with the fur parameter. Wang Ruihua 16 et al analyzed the sublingual vessel and blood routine of patients with heart failure by using the intelligent assistant diagnostic system of tongue manifestation of traditional Chinese medicine. The conclusion is that the changes of sublingual vessel in patients with different NYHA grades are related to erythrocyte, platelet and leukocyte and so on, and more closely related to platelet active components.

\section{Future and Prospect}

At present, doctor's judgment of tongue manifestation is still based on visual observation and personal experience. When we recognize different kind of colors, our eyes' physiological structure and psychological activities will work together, then our brain will tell us what color we have seen. That is to say, different people have different physiological structure and psychological activities of eyes ${ }^{17}$. Therefor we must avoid subjective factors and take experiments analysis data as the standard when we do the quantitative description of tongue color. Nowadays, many studies have been carried out on the objectification of tongue manifestation, and some achievements have been made in digital image acquisition technology and tongue manifestation analysis technology. However, further researches are still needed for more accurate technology to meet the medical need. At the same time, many tongue imagine analysis instruments have flooded into the market, but the healthcare industry has not yet established a unified standard, which is not conducive to the standardization of diagnostic criteria. In addition, the price of the instruments has also become a hindrance to the development. Therefore, a price-friendly tongue imagine analysis instruments with advanced technology is more conducive to the study of the digitalization and objectification of the tongue manifestation, and more conducive to the innovation of the tongue inspection, which can promote the inheritance and development of TCM.

\section{References}

[1] Meng Xianyou, Huang Shuiqing. Origin and Development of Tongue Diagnosis of TCM [J]. Liaoning Journal of Traditional Chinese Medicine, 2016; 43(05):946-948.

[2] Yang Zhujie. Application of Tongue Diagnosis in Internal Medicine of Traditional Chinese Medicine [J]. World Latest Medicine Information, 2016; 16(66):194.

[3] Wang Qi. Classification and diagnosis basis of nine basic constitutions in Chinese medicine[J]. Journal of Beijing University of Traditional Chinese Medicine, 2005; (04):1-8.

[4] Li Haiyu, Ding Jingsheng. Professor SHEN Shao-gong's academic thought on tongue diagnosis and treatment [J]. Chinese Journal of Basic Medicine in Traditional Chinese Medicine, 2018; 24(12):1672-1673.

[5] Wang Chunrong. Analysis of experience of pediatric tongue diagnosis [J]. Shanxi Journal of Traditional Chinese Medicine, 2018; 34(01):1-3.

[6] Lu Dawei, Xi Shengyan. Experience and proved cases of WAN Yan-hui in treating exogenous damp-heat diseases [J]. China Journal of Traditional Chinese Medicine and Pharmacy,2 017; 32(09):4049-4052.

[7] Chen Rongjin, Du Yaqi. Analysis of Tongue Diagnosis in Shanghan Zabing Lun

[J/OL]. Henan Traditional Chinese Medicine, 2019; (01):9-13.

[8] Li Junchuan. Tongue Diagnosis in Shangha Zabing Lun[J]. Hubei Journal of Traditional Chinese Medicine, 1980; (5):12-14

[9] Li Naimin. Tongue Diagnosis of Traditional Chinese Medicine [M].Beijing: Academy Press, 1995.

[10] Deng Tietao. Diagnostics of Traditional Chinese Medicine [M].Shang Hai: Shanghai Scientific \& Technical Publishers, 2001; 18-19.

[11] Ma Xuxiang, Li Yongzhi. Application of Information Technology in Tongue Diagnosis in Syndrome Diagnosis and Therapeutic Evaluation[J]. Chinese Journal of Basic Medicine in Traditional Chinese Medicine, 2018; 24(12):1716-1719.

[12] Shi Qiang, Tang Weichang. Light Source Selection in Tongue Information Objectification [J]. Journal of Shanghai University of Traditional Chinese Medicine, 2004; (02):39-41. 
[13] Li Bocong, Huang Qimei. CIELAB Color Space Based Classification of Tongue Colors [J]. Modernization of Traditional Chinese Medicine and Materia Materia-World Science and Technology, 2007; (03):28-32+54.

[14] Shen Lansun, Wang Aimin. Image Analysis for Tongue Characterization[J]. Acta Electronica Sinica, 2001; (S1):1762-1765.

[15] $\mathrm{Xu} J$ Jie, $\mathrm{Xu}$ Jiatuo. Exploration on correlation between glycometabolism and digital tongue picture in diabetic patients
[J]. Shanghai Journal of Traditional Chinese Medicine, 2014; 48(11):11-13+17.

[16] Wang Ruihua, Li Ya nfen. Relationship between Sublingual Collaterals Changes and Blood Components Content in Patients with Chronic Heart Failure[J]. Chinese Journal of Integrative Medicine on Cardio-/Cerebrovascular Disease, 2018; 16(07):833-840.

[17] Han Xiaowei. Study on Key Technologies of Color Image Processing [D]. Northeastern University,2 005. 